\title{
Ensayo terapéutico con dosis oral única de pamoato de pirantel en las infecciones por Enterobius vermicularis y Ascaris lumbricoides
}

\begin{abstract}
DRES. ISABEL NOEMI *, HERNAN REYES *, Y OSCAR ABELIUK * Y SRTAS. GABRIELA MAURO ** Y BERBELI ASTORGA **
\end{abstract}

En 1966, Austin y col. ${ }^{1}$ sintetizaron un nuevo derivado de la pirimidina, el pirantel **", y bajo forma de tartrato lo ensayaron en animales domésticos, comprobando su fuerte acción antihelmíntica frente a numerosas especies de nematodes intestinales. Nuevos ensayos en animales permitieron demostrar que con una sola dosis oral el fármaco resultaba particularmente efectivo contra Strongyloidea, Ascaroidea y Oxytridae; siendo, por el contrario, poco activo para eliminar las infecciones por Trichuridae y por cestodes ${ }^{2},{ }^{3},{ }^{4}$. En 1968 Cornwell y Jores ${ }^{5}$ lo utilizaron en perros, bajo forma de pamoato, comprobando que mantiene su buen rendimiento y presenta mejor tolerancia para los animales. En ese mismo año, Guarniera y col. ${ }^{6}$ introducen el pamoato de pirantel en medicina humana al tratar con dosis única a personas infectadas por Enterobius vermicularis y establecen que su efecto terapéutico supera, incluso, al del pamoato de pirvinio, la droga de elección hasta ese momento.

Desde entonces, las contribuciones de numerosos autores han permitido comprobar el alto rendimiento terapéutico del pamoato de pirantel contra algunas nematodiasis intestinales del hombre, en particular contra Ascaris lumbricoides y Enterobius vermicularis $\left({ }^{7}\right.$ a $\left.{ }^{28}\right)$. En estas dos infecciones las cifras de curación varían entre 80 y $100 \%$ para la ascariasis, y 85 y $96 \%$ para la enterobiasis. Los mismos estudios revelan, además, una excelente tolerancia de los pacientes frente al medicamento en dosis total única de $10 \mathrm{mg}$ por $\mathrm{Kg}$ de peso corporal.

Con el propósito de entregar un aporte más a estas experiencias, se presenta el resultado ob-

* Unidad de Parasitología, Departamento de Medicina Experimental, Facultad de Medicina, Sede Santiago-Oriente de la Universidad de Chile.

* Laboratorio de Parasitología, Hospital L. Calvo Mackenna y Laboratorio de Parasitología. Hospital del Salvador, Servicio Nacional de Salud, Santiago.

*** Trans-1,4,5,6-tetrahidro-1-metil-2 [2 (2-tienil)] vinil pirimidina hidrogenada. tenido con pamoato de pirantel en dosis oral única en pacientes con enterobiasis y ascariasis, procedentes de los Consultorios Externos de los hospitales L. Calvo Mackenna y del Salvador, en Santiago. Este estudio fue realizado en el período julio a diciembre de 1974 .

Material y método. Se citó por escrito a los pacientes ambulatorios que fueron encontrados positivos a huevos de $E$. vermicularis y $A$. lumbricoides en los exámenes parasitológicos efectuados en los laboratorios respectivos de los hospitales señalados. El diagnóstico de enterobiasis se realizó mediante el método de Graham ${ }^{27}$, consistente en la frotación anal con papel celofán engomado, antes del aseo matinal, por 5 días consecutivos. Para la ascariasis se procedió al examen de 3 muestras fecales, un día por medio, según el método de Teleman modificado ${ }^{28}$.

Concurrieron y completaron todos los requisitos del tratamiento y de los controles clínicoparasitológicos posteriores, 75 pacientes con enterobiasis (65 niños entre 1 y 14 años de edad y 10 adu?tos) y 13 pacientes con ascariasis (todos los cuales fueron niños entre 1 y 14 años de edad); de estos pacientes con ascariasis hubo 4 que presentaron, además, infección por Trichuris trichiura. Otro grupo numeroso de personas recibió tratamiento, pero no completó la totalidad de los controles y ha sido excluido de los resultados.

Cada uno de estos pacientes recibió tratamiento en dosis oral única de pamoato de pirantel equivalente a $10 \mathrm{mg}$ por $\mathrm{Kg}$ de peso corporal, utilizando una suspensión oral con sabor a caramelo que contenía $50 \mathrm{mg}$ de droga base por $\mathrm{ml}^{2}$. En los casos de enterobiasis se efectnó también el tratamiento simultáneo del grupo familiar y se insistió en la adopción de las medidas de higiene personal y del hogar que son necesa-

** "Combantrin", Pfizer. 
rias para la efectiva erradicación de esa parasitosis ${ }^{29}$

Todos los pacientes fueron sometidos a control clínico a los 7,14 y 21 días después del tratamiento, que consistió principalmente en interrogar sobre la forma de cumplir la prescripción medicamentosa, la presencia de síntomas de intolerancia, o la ejecución correcta de las medidas complementarias en los casos de enterobiasis. De igual modo, se efectuó control parasitológico de cada caso a los 7 y 30 días después del tratamiento, realizado con los mismos métodos y número de muestras que para el diagnóstico inicial.

Para la valoración del efecto terapéutico de la droga, sólo se consideró como curadas a aquellas personas en quieres todos los exámenes de las 2 series de control parasitológico fueron negativos.

Resultados. En la Tabla I aparecen los resultados obtenidos en el tratamiento de la en-

\section{T A B L A 1}

RESULTADO DEL TRATAMIENTO CON PAMOATO DE PIRANTEL EN 75 PACIENTES CON ENTEROBIASIS Y 13 PACIENTES CON ASCARIASIS SANTIAGO, 1974

\begin{tabular}{|c|c|c|c|c|c|c|}
\hline \multirow{3}{*}{ Edad (años) } & \multicolumn{3}{|c|}{ Enterobiasis } & \multicolumn{2}{|c|}{ Ascariasis } & \multirow{3}{*}{ Tratam } \\
\hline & & Desp. & Trato & & Desp. & \\
\hline & Casos & $(-)$ & $(+)$ & Casos & $(-)$ & \\
\hline $\begin{array}{l}\text { 1a1d - 2* } \\
\text { (Lact. Mayor) }\end{array}$ & ) & 1 & 1 & 2 & 2 & 0 \\
\hline $\begin{array}{l}\text { 2ald - } 6 \\
\text { (Pre-escolar) }\end{array}$ & 20 & 16 & 4 & 4 & 3 & 1 \\
\hline $\begin{array}{l}\text { 6a1d - } 14 \\
\text { (Escolar) }\end{array}$ & 43 & 36 & 7 & 7 & 7 & 0 \\
\hline $14 \mathrm{a} 1 \mathrm{~d}-20$ & 1 & 1 & 0 & 一 & - & - \\
\hline $21-30$ & 6 & 6 & 0 & - & - & - \\
\hline $31-40$ & 3 & 3 & 0 & - & - & - \\
\hline TOTAL & 75 & 63 & 12 & 13 & 12 & 1 \\
\hline
\end{tabular}

" "a" y "d": año y día, respectivamente.

T A B L A I I

EFECTO ANTIHELMINTICO DEL PAMOATO DE PIRANTEL EN DOSIS ORAL UNICA EN PACIENTES AMBULATORIOS. SANTIAGO 1974

\begin{tabular}{lccc}
\hline & $N^{o}$ personas & $N^{\circ}$ personas & Exito \\
& Tratadas & Curadas & Terapéutico \\
\hline E. vermicularis & 75 & 63 & $84,0 \%$ \\
A. lumbricoides & 13 & 12 & $92,3 \%$ \\
\hline
\end{tabular}

terobiasis y la ascariasis, distribuidos segín la edad de los pacientes. Dado que la mayoría de los casos correspondió a niños hemos utilizado el esquema de edades pediátricas que es habitual en nuestro medio, por cuanto permite agruparlos de acuerdo a su desarrollo sicomotor y de hábitos y actitudes que pudieran incidir en la prevalencia de estas parasitosis; los adultos figuran agrupados en décadas, como es costumbre en estos casos.

De los datos expuestos en la Tabla I puede ser deducido que hubo un porcentaje inferior de fracasos terapéuticos en la infección por 4. lumbricoides ( 1 de 13 casos tratados: 7,7\%), que en los infectados por $E$. vermicularis (12 de 75 casos tratados: 16,0\%). Además, en los casos de enterobiasis parece insinuarse una relación inversa entre el porcentaje de fracasos y la edad de los pacientes, ya que a menor edad el porcentaje es mayor: $18,2 \%$ entre lactantes mayores y pre-escolares y $16,3 \%$ en los escolares; sin embargo, las cifras absolutas son demasiado pequeñas como para establecer esta relación con seguridad. También es interesante anotar que los fracasos sólo aparecieron entre los niños $\mathrm{v}$ no en adultos.

A los datos expresados en dicha Tabla, cabe agregar que de los 4 casos de infección mixta por áscaris y tricocéfalos, los controles parasitológicos revelaron que todos ellos se hicieron negativos para $A$. lumbricoides pero 3 persistieron positivos a huevos de $T$. trichiura.

Respecto a los índices de curación para ambas parasitosis, figuran resumidos en la Tabla II. Correspondieron al $84,0 \%$ en las infecciones por E. vermicularis y al $92,3 \%$ en las por $A$. lumbricoides.

Finalmente, es de destacar que la droga fue en general muy bien tolerada por los pacientes, existiendo apenas 5 casos $(5,7 \%)$ con leve intolerancia, cuya principal manifestación consistió en sensación nauseosa en 2 casos, dolor abdominal en 1 , nerviosismo en 1 y diarrea en 1 .

Discusion. Los resultados obtenidos revelan que el rendimiento terapéutico del pamoato de pirantel, administrado en dosis oral única de 10 $\mathrm{mg}$ por $\mathrm{Kg}$ de peso corporal, alcanzó al $92,3 \%$ en ascariasis y al $84,0 \%$ en enterobiasis, valores que son coincidentes con los comunicados por numerosos autores en el extranjero $(6,7,8,9,10,12$, $13,16,17,18,19,20,21,23,24,26)$. Este hecho, unido a la buena tolerancia que los pacientes exhibieron a la droga, a la facilidad de su administración en una sola dosis oral y a la fácil aceptación de ella, incluso por niños de corta edad, en virtud de la forma farmacéutica edulcorada que fue usada, hablan de su alto valor en el tratamiento de estas dos parasitosis.

Por el contrario, y aun cuando el número de casos tratados es demasiado pequeño para 
poderlo asegurar con precisión, el pamoato de pirantel parece carecer de efecto terapéutico importante contra la tricocefalosis, a la dosis usada por nosotros, tal cual ha sido determinado con mayor propiedad por autores de otros paí$\operatorname{ses}^{2},{ }^{3},{ }^{4}$.

En relación a los fracasos del tratamiento, interesa comentar que ellos se presentaron cen mayor frecuencia en la enterobiasis y en los niños de menor edad. Ambos hechos tienen su explicación en la alta transmisibilidad de esta infección debido a la dispersión de huevos inmediatamente infectantes que los individuos parasitados van provocando. Algunos autores enfatizan el "ambiente oxyurótico" que se crea en torno a estos pacientes y que tiene su máxima expresión en el núcleo familiar, en los internados $\mathrm{y}$ otros lugares de estrecha convivencia ${ }^{30},{ }^{31},{ }^{32}$. Así se determinan fuertes posibilidades de rápida reinfección si no se adoptan las medidas correctivas del caso, y que será mayor mientras menos desarrollados estén los hábitos de higiene individual, como ocurre en los niños de más corta edad. Fue para obviar este inconveniente que en cada caso de enterobiasis se exigió el tratamiento simultáneo del grupo familiar y la aplicación de las medidas pertinentes de higiene personal y de la vivienda, lo cual pudo no haber sido cumplido siempre en buena forma. Distinto es el caso de la ascariasis que, por el ciclo biológico del parásito, requiere de mayores condiciones epidemiológicas y de mayor tiempo para que se establezcan infecciones detectables.

Sin duda, la exigancia de todos estos requisitos para determinar el valor terapéutico de la droga en cuestión, más el cumplimiento de las series completas de cada control parasitológico, castiga los porcentajes de curación inmediata que hubiese sido posible caicular. Sin embargo, ello permite precisarlos en cifras más reales y útiles. Del mismo modo, al efectuar este estudio en pacientes de Consultorio Externo se pretendió también remedar la forma habitual en que el médico clínico debe actuar. Habría sido más fácil y rápido tomar alguno de los jardines infantiles o internados del Area de Salud Oriente para reunir en menor tiempo un buen número de casos de enterobiasis, o alguna de las poblaciones suburbanas para los casos de ascariasis, pero nos parecieron situaciones epidemiológicas particulares del problema y no la realidad clínica normal para el médico tratante.

\section{RESUMEN}

Mediante dosis oral única de $10 \mathrm{mg}$ por $\mathrm{Kg}$ de peso corporal se tratan con pamoato de pirantel 75 casos de enterobiasis y 13 casos de ascariasis diagnosticados mediante frotis anal seriado
(5 muestras) y por examen seriado de heces ( 3 muestras), respectivamente. Todos los pacientes fueron sometidos a control parasitológico, en igual forma que para el diagnóstico inicial, a los 7 y 30 dias después del tratamiento. Considerando como curadas sólo a las personas cuyos exámenes fueron persistentemente negativos, se obtuvo un indice global de curación parasitológica correspondiente a $92,3 \%$ en ascariasis y a $84,0 \%$ en enterobiasis. La tolerancia de la droga fue excelente y sólo el 5,7\% de los casos presentó leves y fugaces molestias atribuibles a ella.

\section{SUMMARY}

Seventy five patients with enterobiasis and thirteen with ascariasis were treated with a single oral dose of pyrantel pamoate, $10 \mathrm{mg}$ per body weight. Infections diagnosis was made through 5 serial Graham perianal scotch-tape samples and examination of 3 serial stool specimens respectively. All patients were submitted for parasitological control with the same diagnostic procedures at 7 and 30 days after treatment. Only those people who showed both controls to be negative were considered as cured. Parasitological cure rates were 92,3\% for ascariasis and $84,0 \%$ for enterobiasis. Only $5.7 \%$ of patients exhibited mild and short side effects with the drug used.

\section{REFFRENCIAS}

1.-Austin, W.C., Courtney, W., Danilewicz, J.C., Morgan, D.H., Conover. L.H., Howes, H.L. Jr., Lanch, J.E., Mc Farland, J.W., Cornwell, R.L. and Theodorides, V.J. Pyrantel tartrate. A new anthelmintic effective against infections of domestic animals. Nature, 212: 1273-1274, 1966.

2.-Cornwell, R.L. Controlled laboratory trials in sheep with anthelmintic pyrantel tartrate. Vet. Rec., 79: 590-595, 1966.

3.-Cornwell, R.L. Field trials in sheep with the anthelmintic pyrantel tartrate. Comparative trials in the prevention of Nematodirus infection in lambs. Vct. Rec., 79: 626-629, 1966.

4.-Howes, H.L. and Lynch, J.E. Anthelmintic studies with pyrantel. I. Therapeutic and prophylactic efficacy against the enteral stages of various helminths in mice and dogs. J. Parasitol., 53: 10851091, 1967.

5.-Cornwell, R.L. and Jones, RM.. Anthelmintic activity of pyrantel pamoate against Ancylostoma caninum in dogs. J. Trop. Med. Hyg., 71: 165-166, 1968.

6.-Guarniera, D., Leonardi, G., Ricci, P. e Ceccarelli, $G$. Il pamoato di pirantel nella terapia delle infestazione da E. vermicularis. Nota preliminare. Ped. Int. (Roma), 18: 225-262, 1968. 
7.-Bumbalo, T.S., Fugazzotto, D.J. and Wyczalek, J. $H$. Treatment of enterobiasis with pyrante! pamoate. Amer. J. Trop. Med. and Hyg., 18: 50-52, 1969.

8.- Amato Neto, V., Levi, G.C. e Campos, L.L. Observacoes sobre a atividade anti-helmintica do pamoato de pirantel. I. Tratamento da ascaridiase. Rev. Inst. Med. Trop. S. Paulo, 12: 207-210, 1970.

9.-Levi, G.C., Amato Neto, V., Ruano, A.C., Vasconcelos, A.J. de, e Campos, $R$. Observacoes sobre a atividade antihelmintica do pamoato de pirantel. II. Tratamento da enterobiase. Rev. Inst. Med. Trop. S. Paulo, 12: 343-346, 1970.

10.-Desowitz, R.S., Bell, T., Williams, J., Cardines, R. and Tamarua, $M$. Anthelmintic activity of pyrantel pamoate. Amer. J. Trop. Med. and Hyg., 19: $775-778,1970$.

11.-Botero, $D$. and Pérez, A. Clinical evaluation of a new drug for the treatment of Ancylostomosis. Amer. J. Trop. Med. and Hyg., 19: 471-475, 1970.

12.-Bell, W.J. and Nassif, S. Comparison of pyrantel pamoate and piperazine phosphate in the treatment of ascariasis. Amer. J. Trop. Med and Hyg., 20: $584-588,1971$.

13.-Cervoni, W.A. anıl Oliver González, J. Clinical evaluation of pyrantel pamoate in helminthiasis. Amer. J. Trop. Med. and Hyg., 20: 589-591, 1971.

14. - Botero, D. Tratamiento de uncinariasis con Combantrin (Pfizer), (Trabajo preliminar presentado en la Mesa Redonda sobre pamoato de pyrantel), VII Congr. Brasil. Med. Trop., Manaus, febrero 1971.

15.-Botero, D. Tratamento de Necator americanus e Ascarts lumbricoides com Combantrin (Pfizer). Trabalho preliminar apresentado na XX Conv. Nac. Soc. Colomb. Gastroent., Bucaramanga, Julho 1971.

16.-Pennisi, L. and Lazzara, A. Pyrantel pamoate in the therapy of Enterobius vermicularis infestation. J. Parasitol., 57: 27-28, 1971.

17.- Sanati, A. and Ghadirian, E.G. Treatment of enterobiasis with pyrantel pamoate in Iran. I. Trop. Med. Hyg., 74: 160-161, 1971.

18.-Villarejos, V.M., Arguedas-Gamboa, J.A., Eduarte. $E$. and Swartzwelder, J.C. Experiences with the anthelmintic pyrantel pamoate. Amer. J. Trop. Med. and Hyg., 20: 842-845, 1971.

19.-Rodrigues, L.D. e Martirani, I. Pamoato de pyrantel, novo e eficaz tratamento de necatoriase e de outras parasitoses intestinais. Rev. Assoc. Med. Brasil, 17: 363-366, 1971.

20.-Katz, N., Zicker, F., Chávez, A. e Antunes, C. $M . F$. Estudos clínicos com pamoato de pirantel nas parasitoses intestinais. Rev. Inst. Med. Trop. S. Paulo, 14: 212-221, 1972.
21.-Levi, G.C., Amato Neto, V., Konichi, S.R. e Stefani, H.N.V. Tratamento da ascaridiase em zona rural. Estudo comparativo entre as atividades de doses únicas de hexa-hidrato de piperazina, tetramisole e pamoato de pirantel. Rev. Inst. Med. Trop. S. Paulo, 14: 392-396, 1972.

22.-Botero, D. y Castaño, A. Tratamiento comparativo de uncinariasis y ascariasis con pamoato de pirantel, hidroxinaftoato de befenio y tetracloroetileno. II Congr. Colomb. Med. Int., Bogotá, 1972.

23.- Ghadirian, E., Sanati, A. Misaghian, G. and Jossefi, $A$. Treatment of ascariasis with pyrantel pamoate in Iran. J. Trop. Med. Hyg. 75: 195-197, 1972.

24. Rim, H.J. and Lim, J.K. Treatment of enterobiasis and ascariasis with Combantrin (Pyrantel pamoate). Trans. Roy. Soc. Trop. Med. Hyg., 66: 170$175,1972$.

25.-Botero, D. and Castıño, A. Estudo comparativo do pamoato de pirantel, hidroxinaftoato de befenio $\mathrm{e}$ tetraclorotileno no tratamento das infestacoes por Necator americanus. Amer. J. Trop. Med. and Hyg., 22: 45-51, 1973.

26. Chanco, P.P., Cabe, E. and Vidad, M.J. The efficacy of pyrantel pamoate in the treatment of ascariasis (A comparative study with piperazine and tetramisole). J. Philipp. Med. Assoc., (fotocopia sin indicar otros datos de referencia).

27.-Graham, D.F. A device for the diagnosis of enterobius infection. Amer. J. Trop. Med., 21: 150$161,1941$.

28.--Neghme, A., Silva, R. y Artigas, J. El laboratorio en el diagnóstico de la amibiasis intestinal. Bol. Chile. Parasit., 10: 66-71, 1955.

29. Atías, A. Esquemas terapéuticos de las enteroparasitosis más frecuentes en Chile. Bol. Chile, Parasit., 26: 36-45, 1971.

30.-Cuevas, R., Schenone, H., Silva, R., Galdames, M., Inzunza, E. y Romero, E. Frecuencia de infección por Enterobius vermicularis en un internado. Bol. Chile, Parasit., 24: 121-123, 1969.

31.-Schenone, H., Arias, B., Galdames, M., Subiabre, V., Cuevas, R., Inzunza, E., Romero, E. y Jiménez, $M$. Rendimiento de los exámenes seriados en el diagnóstico de laboratorio de la infección por $E n-$ terobius vermicularis. Bol. Chile. Parasit., 25: 113 . $117,1970$.

32.--Atias, A., García, J. y Leyton, O. Estudio de la oxyuriasis en un grupo de alumnos de un internado. Bol. Hosp. S. J. Dios (Stgo.), 20: 103-105, 1973.

AGRADECIMIENTOS: Al Departamento Médico de Laboratorios Pfizer de Chile por proporcionar el medicamento, fotocopias de apartados y las fichas para los controles clínico-parasitológicos. A Marfa A. Tobar y María E. Olavarría, preparadoras técnicas de los laboratorios de Parasitología, Hospital L. Calvo Mackenna y Hospital del Salvador, Santiago. 